\title{
Exposure to Oil during Meiosis Results in Alterations in Meiotic Chromosomes that are Similar to Age-Related Changes in the Nematode Caenorhabditis elegans
}

\section{Paul Goldstein}

Department of Biological Sciences, University of Texas at El Paso, El Paso, USA.

Email: pgoldste@utep.edu

Received June $16^{\text {th }}, 2010$; revised June $26^{\text {th }}, 2010$; accepted July $16^{\text {th }}, 2010$.

\begin{abstract}
Exposure of young C. elegans nematodes to three different concentrations of oil resulted in changes in the meiotic chromosomes, nucleus, nucleolus, and nuclear envelope associations. Such alterations decreased the viability and fertility of this organism which was used as a biological model. The morphological changes in the "young" group were similar to nematodes that were senescent and post-reproductive. Comparison of meiotic chromosomes at the pachytene stage of meiosis from young, old, and oil-exposed wild-type hermaphrodites were made following three-dimensional electron microscopy reconstruction of serial ultrathin sections. Age-related and oil-exposure related changes included: 1) Induced condensation of chromatin with increased variance in length of chromosomes; 2) Changes in nuclear and nucleolar volume; 3) Increased density of the nucleoplasm; and 4) Absence of Disjunction Regulator Regions, resulting in the loss of control of the segregation of the X-chromosome into gametes during meiosis. Abnormal clustering of the telomeric ends of the chromosomes was present on the nuclear envelope affecting the segregation of the chromosomes during meiosis.
\end{abstract}

Keywords: Oil, Age, Chromosome, Synaptonemal Complex

\section{Introduction}

Cytogenetic studies in many organisms have shown that there is a change in chromosome number or an increase in structural chromosome alterations associated with aging [1]. Such changes would account for the cellular deterioration characteristic of senescent tissues since the production of gene products would be altered. It is of significance that such changes in normal structure and function can be induced by exposure to oil [2].

In the present study, the free-living nematode Caenorhabditis elegans, was exposed to varying concentrations of oil and used as a biological model to characterize changes associates with such exposure. This nematode is ubiquitous and is found in virtually all types of environments, including marine. It reproduces primarily as a self-fertilizing hermaphrodite, but a small number of morphologically distinct males $(0.3 \%)$ are also present in the population [3]. The adult hermaphrodite has a pair of ovaries, 810 nongonadal nuclei and five pairs of autosomes with two X chromosomes (2A:XX). The pachytene nuclei are arranged peripherally around a central rachis (Figure 1). The adult male has a single testis, 970 nongonadal nuclei, and five pairs of autosomes but only a single $\mathrm{X}$ chromosome (2A:XO) [4]. Males arise from gametes that have been produced after X-chromosome nondisjunction, thus, the two sexes experience an unequal number of $\mathrm{X}$ chromosomes. They must compensate for this state of aneuploidy and develop mechanisms for gene expression and dosage compensation [5]. Distinct structures on the chromosomes called "Disjunction Regulator Regions" control the separation of the X chromosome during meiosis. Such separation is essential in order to produce functional gametes that have the correct number of chromosomes [6].

The synaptonemal complex (SC) is a tripartite, proteinaceous structure that is found between paired chromosomes during the pachytene stage of meiosis. It has 


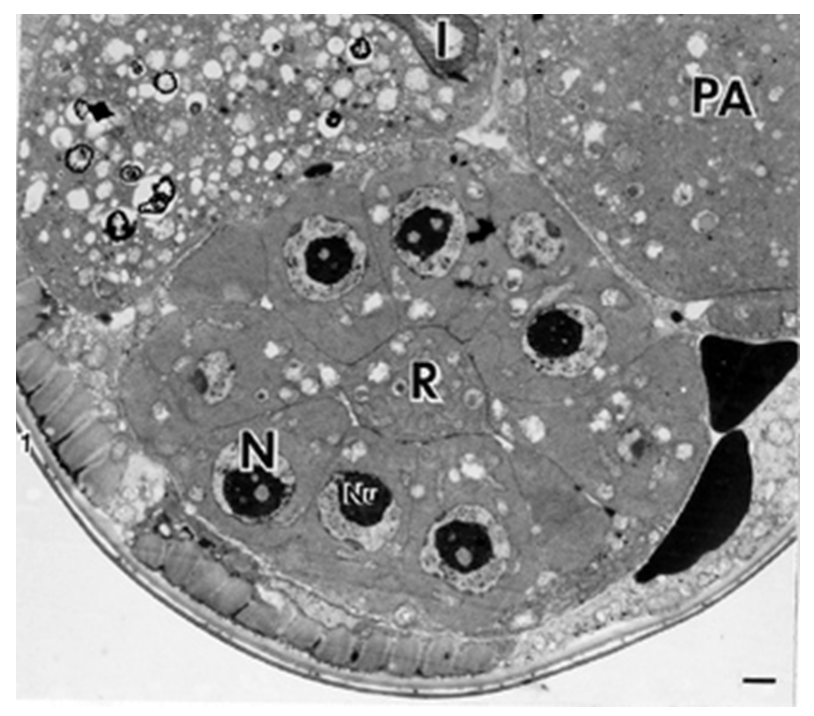

Figure 1. Cross-section of the gonad from $C$. elegans. The meiotic nuclei $(N)$ of the oocytes at the pachytene stage of meiosis are arranged peripherally around a central rachis (R). Nucleolus (Nu) Proximal arm (PA) of the gonad. Bar equals $0.5 \mu \mathrm{m}$.

been highly conserved throughout evolution and occurs in a wide variety of organisms [7]. Its presumed role is twofold: 1) Maintenance of proximity of homologous chromosomal segments, whereby the axial cores of the homologs become the lateral elements of the SC [8]; and 2) Regulation of ordered meiotic disjunction in which case the $\mathrm{SC}$ is maintained at the chiasma [9]. Irregular nondisjunction of the chromosomes at anaphase I may be a reflection of the pairing pattern at pachytene such that multivalent associations, as in polysomies and duplications, yield complex configurations that inhibit segregation [10]. In systems in which normal bivalent formation has been altered by the presence of chromosomal aberrations, the resultant univalents do not show ordered segregation patterns, and the resultant gametes are aneuploid.

In $C$. elegans, there are six SCs per germline nucleus. The XX hermaphrodite has five SCs associated with the autosomal bivalents and one $\mathrm{SC}$ for the $\mathrm{XX}$ bivalent. The $\mathrm{XO}$ male has only five SCs since the univalent X chromosome remains heterochromatic during pachytene $[11,12]$. The tripartite SC consists of two lateral elements and a striated central element with a distance between axes of approximately $100 \mathrm{~nm}$. One end of the SC is attached to the inner nuclear envelope while the other end remains free in the nucleoplasm [12]. There is no evidence of the configuration known as the "bouquet" which facilitates homologous pairing via restricted chromosome movement [13], thus the ends of the chro- mosomes are randomly attached around the nuclear envelope. In addition, there are no recombination nodules present in $C$. elegans although they have been described in other organisms [14].

Disjunction Regulator Regions (DRR) were first described in $C$. elegans $[11,12]$. Statistical analysis of the occurrence of DRRs and the frequency of X-chromosome nondisjunction support the observation that there is a correlation between the presence of DRRs and normal disjunction of the $\mathrm{X}$ chromosome [6], which is required for the production of functional gametes.

The American Petroleum Institute's Manifesto on Crude Oil (CAS \# 8002-05-09; (a.k.a. Sweet Crude, Arab Medium, West Texas Inter-Cushing, Earth Oil, Petroleum Oil, Rock Oil, Zafiro) defines the composition of Crude Oil as "A complex combination of hydrocarbons". It consists predominantly of aliphatic, alicyclic and aromatic hydrocarbons. It may also contain small amounts of nitrogen, oxygen and sulfur compounds. Specifically Crude Oil (CAS \# 8002-05-9) contains Benzene, Butane, N-Hexane, Isopentane, Pentane, and Stoddard Solvent. Benzene is a known human carcinogen and is identified by NTP, OSHA, and IARC as a Group 1 carcinogen. This category encompasses light, medium, and heavy petroleums, as well as the oils extracted from tar sands. Along with the numerous oil spills that are known, there are also significant leaks and seepages into the environment that are not reported that are equally hazardous to the environment and negatively affect the ecosystem. As early as 1982, cytogenetic effects, including mitotic sister chromatid breaks and chromosome damage, were reported after exposure to oil [2].

This study represents the first examination of the changes in nuclear architecture and the meiotic chromosomes after exposure to oil. Serial ultrathin sections and electron microscopy were used to provide three-dimensional analysis of the relationships of all nuclear components and identify changes correlated to the cytotoxicity of oil.

\section{Materials and Methods}

The C. elegans individuals studied were hermaphrodites received from the Caenorhabditis Genetics Center. The worms were grown in agar plates made with $5 \%, 25 \%$, and $50 \%$ Texas Crude oil in the form of an emulsion which they moved through at all levels of the agar. The plate was seeded with OP50 E. coli bacteria as a food source. In order to obtain worms at specific ages, adult worms were removed from the plates leaving only first stage larvae. Worms were selected at 4-5 days and 8-9 days of age, thus, forming the "young" and "old" groups and were processed for electron microscopy as previously 
described [11,15].

The pachytene karyotypes from analysis of synaptonemal complexes of five early-mid pachytene stage nuclei from worms that were 4-5 days old ("young"), 8-9 days old ("old"), and 5\%, $25 \%$ and $50 \%$ oil-exposed were determined using the Metamorphosis program [16]. Nuclear and nucleolar volumes were also determined using the Metamorphosis program. A total of 25 pachytene karyotypes were analyzed.

\section{Results and Discussion}

Comparison of the synaptonemal complexes from the meiotic chromosomes at the stage of pachytene, revealed significant changes in the chromatin, the nucleus, and its associated components (Table 1). These included: 1) Condensation of chromatin (more condensed in older specimens and those exposed to oil; Figure 2); 2) Decrease in length of the chromosomes due to condensation; 3) Decrease in volume of the nucleus due to changes in the nuclear matrix; and 4) Alterations in the association of the telomeric ends of the chromosomes with the nuclear envelope, such that the normally random attachment of the telomeres was restricted to a small region of the nucleus. This resulted in a clustering of the ends of the chromosomes, which affects their ability to segregate properly into the gametes. In this organism, the clustering of the chromosome ends may promote entanglements which cannot be easily resolved. In addition, the nucleus of the old and oil-exposed individuals, accumulated electron-dense proteinaceous materials, and the nuclear envelope was irregular. These are manifestations of the degradation of the nucleoplasm and protein matrix.

The synaptonemal complexes (SC), which are normally tripartite proteinaceous structures within the meiotic chromosomes, were altered in the ten selected worms exposed to $25 \%$ and $50 \%$, such that the structure of the SCs became indistinct and the lateral elements and the central elements were not discernible (Figure 3). This is compared to normal SCs where the two lateral elements were clearly visible (Figure 4). In addition, there was a $26 \%$ decrease in synaptonemal complex length comparing young worms to those five selected worms exposed to $50 \%$ oil (Figure 5, Table 1). There was a variation in length of plus or minus of two percent from one worm to the next. This may be the result of the abnormal condensation of the chromatin which resulted in altered SC structure. The SC is required for pairing and separation of the chromosomes during meiosis, thus, alteration in its structure may result in decreased viability and fecundity.

The Nuclear Volume decreased by $37 \%$ from the five young worms and $18 \%$ from the five old worms to the five worms selected which were exposed to $50 \%$ oil.

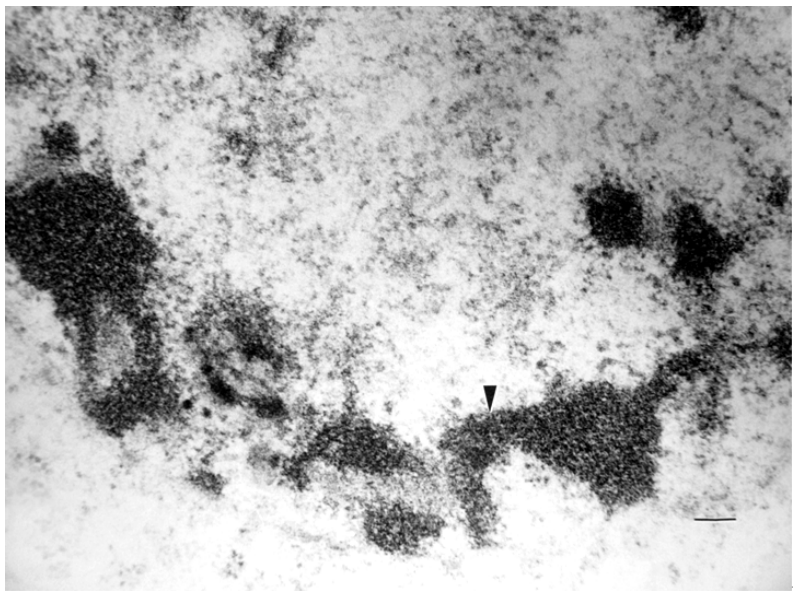

Figure 2. C. elegans 4-5 days old ("young"), exposed to 50\% oil, demonstrate the same condensation in chromatin (arrow) as in senescent worms. Bar equals $0.1 \mu \mathrm{m}$.

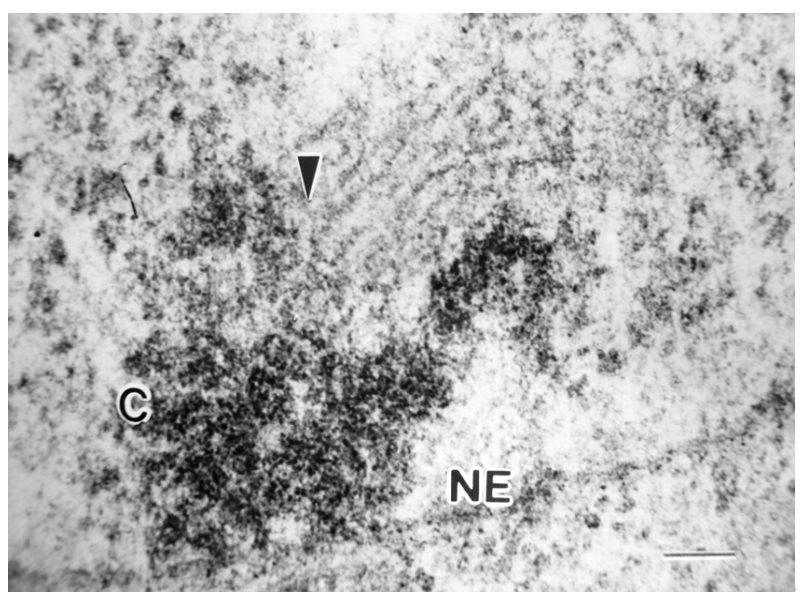

Figure 3. Synaptonemal complexes (arrow) from 4-5 day old ("young") $C$. elegans exposed to $50 \%$ oil are altered in structure. The lateral elements and central element are not discernible. Nuclear Envelope (NE) Chromatin (C) Bar equals $0.2 \mu \mathrm{m}$.

Nuclear volume also decreased $13 \%$ between those worms exposed to $5 \%$ versus $50 \%$ oil (Figure 6, Table 1). This was due to abnormal condensation, i.e. heterochromatization, of the chromosomes and altered protein structure. Heterochromatization is a normal process in senescent cells (those from $C$. elegans that are 8-9 days old) and results in decreased expression of genes. The fact that the same morphology is manifested in 4-5 day old cells ("young") after exposure to $50 \%$ oil, is significant, as it indicates the deterioration and loss of function in the oocytes [17].

Disjunction Regulator Regions (DRR) appeared as decondensed chromatin and were associated with the synaptonemal complex. In C. elegans, they have been shown 
Table 1. Average pachytene chromosome lengths of $C$. elegans wild-type from young, old, and oil-exposed individuals from three different concentrations by reconstruction of synaptonemal complexes. All chromosome lengths are in microns.

\begin{tabular}{|c|c|c|c|c|c|}
\hline $\mathrm{SC} \#$ & $\begin{array}{c}\text { Young } \\
\text { (4-5 days old) }\end{array}$ & $\begin{array}{c}\text { Old } \\
8-9 \text { days old) } \\
\end{array}$ & $\begin{array}{l}\text { Oil-Exposed } \\
(5 \% \text { Oil })\end{array}$ & $\begin{array}{c}\text { Oil-Exposed } \\
(25 \% \text { Oil })\end{array}$ & $\begin{array}{c}\text { Oil-Exposed } \\
(50 \% \text { Oil })\end{array}$ \\
\hline $1 \mathrm{x}$ & 2.5 & 2.4 & 2.4 & 2.2 & 1.9 \\
\hline 2 & 4.5 & 4.1 & 4.1 & 4.0 & 3.8 \\
\hline 3 & 6.0 & 5.5 & 5.3 & 5.0 & 4.7 \\
\hline 4 & 6.2 & 6.0 & 6.0 & 5.4 & 4.7 \\
\hline 5 & 7.9 & 7.1 & 7.0 & 6.8 & 6.5 \\
\hline 6 & $10.4^{\mathrm{a}}$ & $9.1^{\mathrm{a}}$ & $9.0^{\mathrm{a}}$ & $8.0^{\mathrm{a}}$ & $6.0^{\mathrm{a}}$ \\
\hline Total & 37.5 & 34.2 & 33.8 & 31.4 & 27.6 \\
\hline Nuclear Volume $\mu \mathrm{m}^{3}$ & 12.4 & 9.4 & 9.0 & 8.8 & 7.8 \\
\hline $\begin{array}{c}\text { Nucleolar Volume } \\
\mu \mathrm{m}^{3}\end{array}$ & 5.6 & 4.5 & 4.5 & 4.4 & 4.2 \\
\hline$\% \mathrm{Nu}$ Vol. & 45.2 & 47.8 & 50.0 & 50.0 & 58.3 \\
\hline \# DRR & 6 & 3 & 0 & 0 & 0 \\
\hline $\begin{array}{l}\text { Position of NOR } \\
\text { from Telomere }\end{array}$ & $18 \%$ & $25 \%$ & $22 \%$ & $19 \%$ & $37 \%$ \\
\hline
\end{tabular}

(a) Nucleolar Organizer Region (NOR) found on this chromosome; (x) X-chromosome

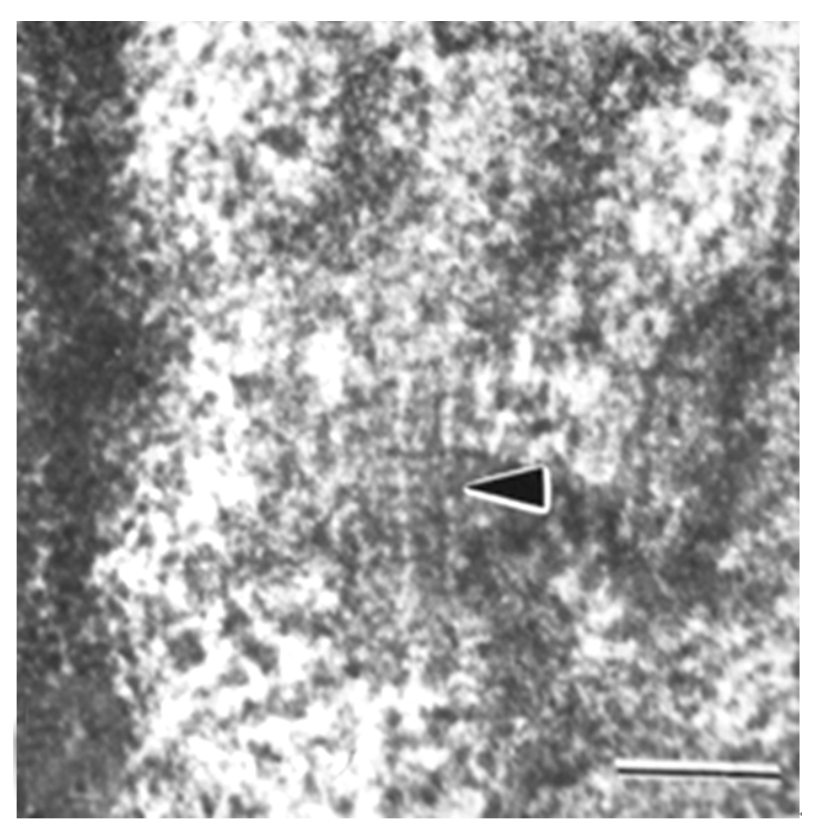

Figure 4. Normal synaptonemal complex from a 4-5 day old ("young") C. elegans comprised of two lateral elements (arrow) and a striated central element that is formed between them. Bar equals $0.1 \mu \mathrm{m}$.

to be responsible for controlled segregation of the Xchromosome during meiosis [6]. The average number of DRRs in nuclei from 4-5 day old nematodes was 6,

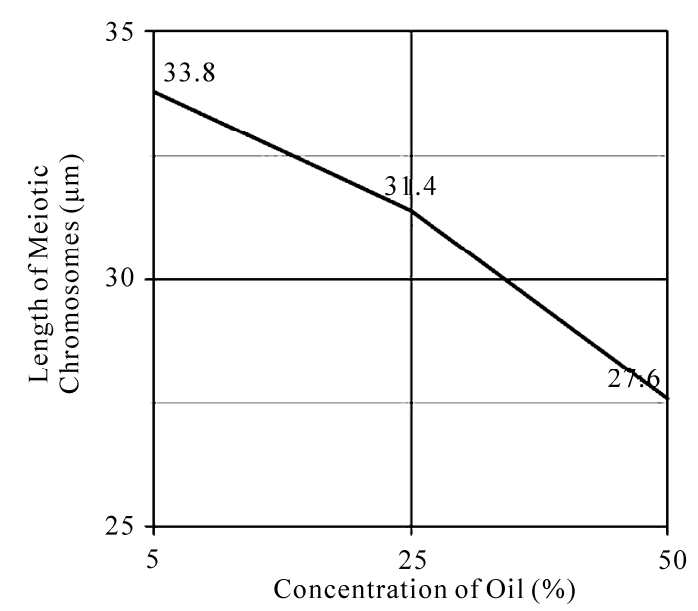

Figure 5. Change in meiotic chromosome length after exposure to varying concentrations of oil.

whereas after they have been exposed to $50 \%$ oil, there were none. Thus, segregation of the X-chromosome was no longer under the proper control and unequal numbers of chromosomes would be distributed to the gametes. Such aneuploid gametes have decreased function.

This study was done in order to determine the effects of exposure to oil on meiotic chromosomes and nuclear components. The nematode $C$. elegans was used as a biological model, however, the mechanisms of meiosis are conserved throughout evolution and these aberrations 


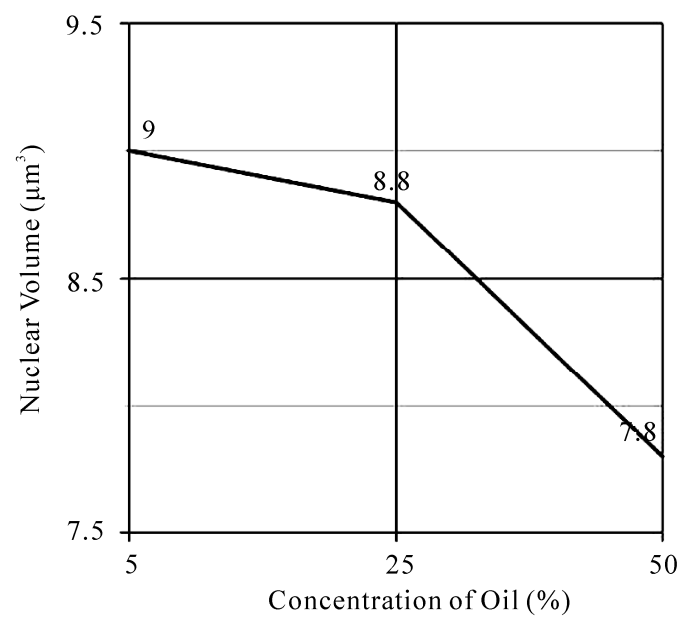

Figure 6. Change in meiotic nuclear volume after exposure to varying concentrations of oil.

may pertain to humans as well.

\section{Acknowledgements}

This publication was made possible in part by Grant \# 5G12RR008124 to the Border Biomedical Research Center (BBRC)/University of Texas at El Paso from the National Center for Research Resources (NCRR)/NIH. The contents of this publication are solely the responsibility of the authors and do not necessarily represent the official views of NIH or NIEHS.

\section{REFERENCES}

[1] D. Kram and E. L. Schneider, "Parental-Age Effects: Increased Frequencies of Genetically Abnormal Offspring," In: E. L. Schneider, Ed., The Genetics of Aging, Plenum Press, NY, 1978, pp. 225-260.

[2] J. Meyne and L. Deaven, "In vivo Cytogenetic Effects of Oil Shale Retort Process Waters," Toxicology, Vol. 24, No. 3-4, 1982, pp. 223-229.

[3] J. Hodgkin, R. Horvitz and S. Brenner, "Nondisjunction Mutants of the Nematode Caenorhabditis elegans," Genetics, Vol. 91, No. 1, 1979, pp. 67-94.

[4] J. Hodgkin, "More Sex Determination Mutants of Caenorhabditis elegans," Genetics, Vol. 96, No. 3, 1980, pp. 649-664.

[5] P. Goldstein, "Aneuploidy in the Normal Life Cycle of the Nematode Caenorhabditis elegans," In: B. Vig and A.
Sandberg, Eds., Aneuploidy, Part A: Incidence and Etiology, Alan R. Liss, New York, 1987, pp. 189-204.

[6] P. Goldstein, “The Synaptonemal Complexes of Caenorhabditis elegans: Pachytene Karyotype Analysis of the Dp1 Mutant and Disjunction Regulator Regions," Chromosoma, Vol. 93, No. 2, 1985, pp.177-182.

[7] D. Von Wettstein, S. W. Rasmussen and P. B. Holm, "The Synaptonemal Complex in Genetic Segregation," Annual Reviews of Genetics, Vol. 18, 1984, pp. 331-413.

[8] P. B. Moens, "The Structure and Function of the Synaptonemal Complex in Lilium longiflorum Sporocytes," Chromosoma, Vol. 28, No. 1, 1968, pp. 1-25.

[9] M. Maguire, "Evidence for Separate Genetic Control of Crossing over and Chiasma Maintenance in Maize," Chromosoma, Vol. 65, No. 2, 1982, pp. 173-178.

[10] S. W. Rasmussen and P. B. Holm, "Mechanism of Meiosis," Hereditas, Vol. 93, No. 2, 1980, pp. 187-216.

[11] P. Goldstein and D. Slaton, "The Synaptonemal Complexes of Caenorhabditis elegans: Comparison of WildType and Mutant Strains and Pachytene Karyotype Analysis of Wild-Type," Chromosoma, Vol. 84, No. 4, 1982, pp. 585-590.

[12] P. Goldstein, "The Synaptonemal Complexes of Caenorhabditis elegans: Pachytene karyotype Analysis of Male and Hermaphrodite Wild-Type and Him Mutants," Chromosoma, Vol. 86, No. 4, 1982, pp. 577-593.

[13] J. Gerton and R. Hawley, "Homologous Chromosome Interactions in Meiosis: Diversity amidst Conversation," Nature Review Genetics, Vol. 6, No. 6, 2005, pp. 477487.

[14] D. Zickler and N. Kleckner, "Meiotic Chromosomes: Integrating Structure and Function," Annual Reviews of Genetics, Vol. 33, 1999, pp. 603-754.

[15] P. Goldstein and K. Lerner, "Electron Microscopic Autoradiographic Analysis: Evidence of RNA Transcription along the Pachytene Chromosomes of C. elegans," Cytobios, Vol. 55, No. 220, 1988, pp. 51-61.

[16] M. Voight and P. Goldstein, "Metamorphosis: ComputerAssisted Rotational Analysis and Interspatial Distribution of Nuclear Structures from Serial Ultrathin Sections," Cytobios, Vol. 51, No. 206-207, 1987, pp. 193-207.

[17] P. Goldstein and M. Curis, "Age-Related Changes in the Meiotic Chromosomes of the Nematode Caenorhabditis elegans," Mechanisms of Aging and Development, Vol. 40, No. 2, 1987, pp. 115-130. 